\title{
MicroRNAs Involved in the Mitogen-Activated Protein Kinase Cascades Pathway During Glucose-Induced Cardiomyocyte Hypertrophy
}

\author{
E Shen, ${ }^{+\dagger}$ Xuehong Diao, ${ }^{*}$ Xiaoxia Wang, ${ }^{\ddagger}$ \\ Ruizhen Chen, ${ }^{\S}$ and Bing $\mathrm{Hu}^{* \dagger}$ \\ From the Department of Ultrasound in Medicine," Shanghai \\ Jiaotong University Affiliated 6th People's Hospital, Shanghai; the \\ Cardiovascular Disease Laboratory, ${ }^{\dagger}$ Shanghai Institute of \\ Ultrasound in Medicine, Shanghai; the Department of Nephrology, \\ Shanghai Jiaotong University Affiliated 6th People's Hospital, \\ Shanghai; and the Shanghai Institute of Cardiovascular Diseases, \\ Zhongshan Hospital, Fudan University, Shanghai, China
}

Cardiac hypertrophy is a key structural feature of diabetic cardiomyopathy in the late stage of diabetes. Recent studies show that microRNAs (miRNAs) are involved in the pathogenesis of cardiac hypertrophy in diabetic mice, but more novel miRNAs remain to be investigated. In this study, diabetic cardiomyopathy, characterized by hypertrophy, was induced in mice by streptozotocin injection. Using microarray analysis of myocardial tissue, we were able to identify changes in expression in 19 miRNA, of which 16 miRNAs were further validated by real-time PCR and a total of 3212 targets mRNA were predicted. Further analysis showed that 31 GO functions and 16 KEGG pathways were enriched in the diabetic heart. Of these, MAPK signaling pathway was prominent. In vivo and in vitro studies have confirmed that three major subgroups of MAPK including ERK1/2, JNK, and p38, are specifically upregulated in cardiomyocyte hypertrophy during hyperglycemia. To further explore the potential involvement of miRNAs in the regulation of glucose-induced cardiomyocyte hypertrophy, neonatal rat cardiomyocytes were exposed to high glucose and transfected with miR-373 mimic. Overexpression of miR-373 decreased the cell size, and also reduced the level of its target gene MEF2C, and miR-373 expression was regulated by $\mathrm{p} 38$. Our data highlight an important role of miRNAs in diabetic cardiomyopathy, and implicate the reliability of bioinformatics analysis in shedding light on the mechanisms underlying diabetic cardiomyopathy. (Am J Pathol 2011, 179:639-650; DOI: 10.1016/j.ajpath.2011.04.034)
MicroRNAs (miRNAs) are a class of endogenous, small, noncoding RNAs that control the target gene expression at the posttranscriptional level. Increasing evidence indicates that miRNAs regulate pathophysiological processes such as cell differentiation, cell proliferation, apoptosis, and organ development. ${ }^{1,2}$ Recent studies have described key roles of miRNAs in cardiovascular biology and heart disease. ${ }^{3,4} \mathrm{~A}$ number of miRNAs have been shown to control the balance between differentiation and proliferation during cardiogenesis; and a variety of heart diseases, such as myocardial ischemia, cardiac fibrosis, cardiac arrhythmias, and heart failure, have been related to aberrant expression of miRNAs. ${ }^{5-7}$ In animal models of hypertrophy, whole arrays of miRNAs have been reported to be dysregulated with respect to the normal heart, thus indicating their common roles in hypertrophy pathogenesis. ${ }^{8,9}$ Nevertheless, the role of miRNAs and their signaling pathways in regulating diabetes-induced cardiomyocyte hypertrophy remain largely unknown.

Diabetic cardiomyopathy occurs independently of coronary artery disease and hypertension, and is a common complication of diabetes. ${ }^{10,11}$ Structural and functional changes in the diabetic heart are characterized by left ventricular hypertrophy (LVH), myocardial fibrosis, diastolic left ventricular dysfunction, and systolic dysfunction. $^{12}$ These pathogenetic changes lead to cardiomyocyte cell loss and reactive cellular hypertrophy, which is the leading cause of diabetes-related morbidity and mortality worldwide. Various molecular pathways and the regulators of hypertrophic response that are responsible for the control of cardiac hypertrophy in diabetes include Rac1, ${ }^{13}$ p300, vasoactive factors, redox-sensitive transcription factors, ${ }^{14}$ TGF1 cascade, insulin and insulin-like growth factor-I (IGFI) signaling, and calcineurin-NFAT3 hypertrophic pathway. ${ }^{15} \mathrm{~A}$ recently published study by

\footnotetext{
Supported by the Shanghai Pujiang Program (grant 09PJ480400).

Accepted for publication April 26, 2011.

E.S. and X.D. contributed equally to this work.

Address reprint requests to Bing Hu, M.D., Department of Ultrasound in Medicine, Shanghai Jiaotong University Affiliated 6th People's Hospital, Shanghai Institute of Ultrasound in Medicine, NO.600, Yi Shan Road, China. E-mail: binghuzz@gmail.com.
} 
Feng et $\mathrm{al}^{16}$ firmly supports the hypothesis that miRNAs plays a key role in cardiomyocyte hypertrophy in diabetes. These investigators confirmed the action of miR-133a by discovering that down-regulation of miR-133a mediates diabetes-induced cardiomyocyte hypertrophy in mice, which resulted in upregulation of the miR-133a regulatory targets SGK1 and IGFR1, two transcription factors associated myocardial hypertrophy. Nevertheless, the signaling pathways that regulate the expression of miR-133a during diabetes-induced cardiomyocyte hypertrophy remain unknown. Thus, further investigation is needed to ascertain whether more novel miRNAs and related networks are associated with cardiac hypertrophy in diabetes.

The primary aim of the present study was twofold: to elucidate the expression of miRNA in cardiac hypertrophy induced by hyperglycemia in mice, and to further understand the role of miRNAs as they relate to diabetic cardiomyopathy. A microarray technique was used to present a comprehensive picture of the expression of miRNAs, and a prediction database was applied to select the target genes of the altered miRNAs. In addition we investigated the functional information of these miRNAs and the related regulatory networks by gene ontology (GO) function and Kyoto Encyclopedia of Genes and Genomes (KEGG) pathway enrichment analysis.

\section{Materials and Methods}

\section{Animal Studies}

Experiments were performed using age- and weightmatched C57/BL6 male mice (8 weeks old, 23 to $25 \mathrm{~g}$ body weight; Sippr-bk Lab Animal Ltd., Shanghai, China). Before and during treatment, all animals were housed in a climate-controlled room with a 12-hour light/ dark cycle. Animals were fed a standard diet and allowed tap water ad libitum. All procedures were performed in accordance with National Institutes of Health $(\mathrm{NIH})$ guidelines (no. 85-23, revised 1996) for the care and use of experimental animals.

Diabetes was induced by a single injection of streptozotocin (STZ, $150 \mathrm{mg} / \mathrm{kg}$ i.p) dissolved in $0.1 \mathrm{~mol} / \mathrm{L}$ citrate buffer, pH 4.3 (Sigma, St. Louis, MO). Nondiabetic control mice received the citrate buffer only. Three day after the injection of STZ or vehicle, blood glucose levels were measured using the Optium Xceed Diabetes Monitoring System (Abbott Diabetes Care, Alameda, CA). Blood glucose content of $\geq 18.6 \mathrm{mmol} / \mathrm{L}$ was chosen as indicating diabetes in the present study. ${ }^{17}$

\section{Cell Culture}

Primary neonatal rat myocytes were isolated from newborn Sprague-Dawley rat heart ventricles by collagenase digestion and cultured as described previously. ${ }^{14}$ In brief, isolated cardiomyocytes were plated onto cell culture dishes at a density of $3.0 \times 10^{4}$ cells $/ \mathrm{cm}^{2}$ and were maintained for 24 hours in Dulbecco's modified Eagle's medium (DMEM). The cells were serum starved 24 hours before use for the experiments. All experiments were performed after 48 hours of incubation with $5.5 \mathrm{mmol} / \mathrm{L}$ (control; NG) or $25 \mathrm{mmol} / \mathrm{L}$ D-glucose (high glucose; $H G)$. A 25-mmol/L quantity of L-glucose was used as an
A

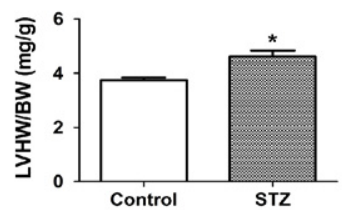

C

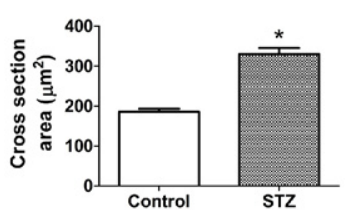

B

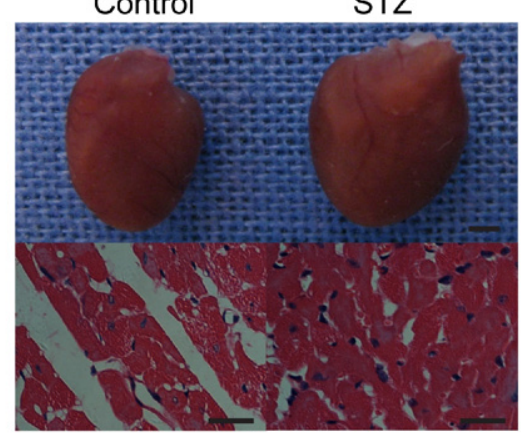

D
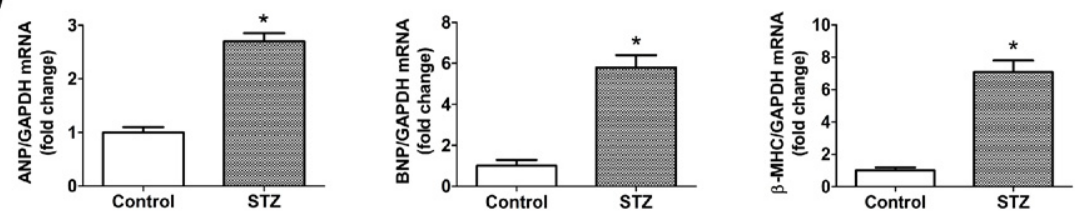

E

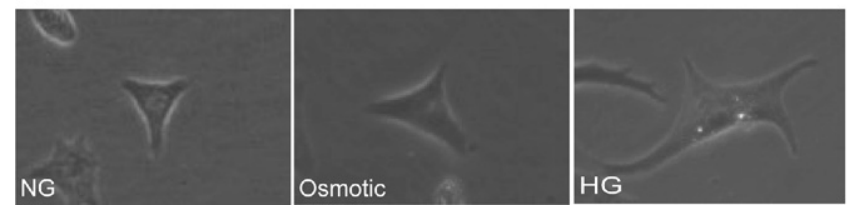

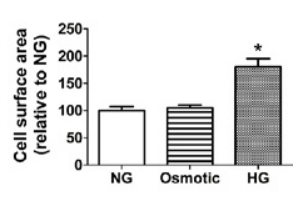

Figure 1. Glucose causes cardiac hypertrophy of diabetic mice and cardiomyocytes hypertrophy of neonatal rat. A: Heart weight to body weight ratios (HW/BW) from diabetic mice ( $n=$ $15)$ and nondiabetic mice $(n=10)$. B: Morphological analysis of hearts from diabetic mice and nondiabetic mice. Scale bar $=1 \mathrm{~mm}$ (top). Hematoxylin-eosin-stained micrographs showing transverse sections of left ventricular myocardium. Original magnification, $\times 40$. Scale bar $=25 \mu \mathrm{m}$ (bottom) C: Measurement of cardiomyocyte cross-sectional area sampled from heart sections ( $n=5$ for each group). D: mRNA expression of ANP, BNP, and $\beta$-MHC in cardiac hypertrophy induced by STZ $(n=5)$. E: Micrograph images of cultured rat neonatal myocytes treated with $25 \mathrm{mmol} / \mathrm{L}$ glucose (HG), 25 $\mathrm{mmol} / \mathrm{L} \mathrm{L}$-glucose (osmotic), or $5 \mathrm{mmol} / \mathrm{L}$ glucose (NG) after 48 hours (left panels). Cell surface areas of more than 100 individualized cells per condition were measured using ImageJ software (right panel). ${ }^{*} P<0.05$ compared with control or NG. 
Table 1. MicroRNA Targets Significant in Hearts of STZ-Induced Diabetic Mice, by GO Identification Number and Name

\begin{tabular}{|c|c|c|c|c|}
\hline GO_id & GO_name & $P$ value & FDR & Enrichment \\
\hline GO:0048009 & $\begin{array}{l}\text { Insulin-like growth factor receptor signaling } \\
\text { pathway }\end{array}$ & 0.000303384 & 0.003028665 & 12.295015576 \\
\hline GO:0007254 & JNK cascade & 2.13953E-05 & 0.000312182 & 11.750222045 \\
\hline GO:0050902 & Leukocyte adhesive activation & 0.000372106 & 0.003627298 & 10.07202492 \\
\hline GO:0007178 & $\begin{array}{l}\text { Transmembrane receptor protein serine/ } \\
\text { threonine kinase signaling pathway }\end{array}$ & 2.8093E-06 & 5.00967E-05 & 7.747711479 \\
\hline GO:0000165 & MAPKKK cascade & 0.000108199 & 0.001246034 & 6.956866934 \\
\hline GO:0030199 & Collagen fibril organization & 5.22196E-06 & 8.91667E-05 & 5.722741433 \\
\hline GO:0008286 & Insulin receptor signaling pathway & 3.26149E-08 & 8.50239E-07 & 4.864330218 \\
\hline $\mathrm{GO}: 0008016$ & Regulation of heart contraction & $2.53336 \mathrm{E}-05$ & 0.0003595 & 4.196677051 \\
\hline GO:0007265 & Ras protein signal transduction & $3.02529 \mathrm{E}-10$ & 1.15234E-08 & 4.046795728 \\
\hline GO:0007179 & $\begin{array}{l}\text { Transforming growth factor beta receptor } \\
\text { signaling pathway }\end{array}$ & 5.34664E-06 & $9.11004 \mathrm{E}-05$ & 3.934384735 \\
\hline GO:0051056 & $\begin{array}{l}\text { Regulation of small GTPase mediated } \\
\text { signal transduction }\end{array}$ & 1.66201E-09 & 5.593E-08 & 3.896914404 \\
\hline GO:0001666 & Response to hypoxia & 7.90691E-09 & 2.32412E-07 & 3.456086983 \\
\hline $\mathrm{GO}: 0030036$ & Actin cytoskeleton organization & $2.41342 \mathrm{E}-13$ & $1.35427 \mathrm{E}-11$ & 3.324334068 \\
\hline GO:0043065 & Positive regulation of apoptosis & $1.89632 \mathrm{E}-06$ & $3.46985 \mathrm{E}-05$ & 3.264082151 \\
\hline GO:0035023 & $\begin{array}{l}\text { Regulation of Rho protein signal } \\
\text { transduction }\end{array}$ & 1.81537E-07 & 4.4.03059E-06 & 3.178066116 \\
\hline GO:0030308 & Negative regulation of cell growth & 5.99755E-08 & 1.50693E-06 & 3.175863714 \\
\hline GO:0007160 & Cell-matrix adhesion & $1.35546 \mathrm{E}-05$ & 0.0 .000210746 & 3.106631064 \\
\hline GO:0007243 & Protein kinase cascade & 9.54633Е-08 & 2.2.3098E-06 & 3.042590862 \\
\hline GO:0007264 & Small GTPase mediated signal transduction & 8.12556E-19 & 7.5993E-17 & 2.988731431 \\
\hline $\mathrm{GO}: 0016055$ & Wnt receptor signaling pathway & $5.97358 \mathrm{E}-10$ & 2.2.17568E-08 & 2.932904984 \\
\hline $\mathrm{GO}: 0006006$ & Glucose metabolic process & 0.000191271 & 0.0 .001987601 & 2.877721406 \\
\hline GO:0007010 & Cytoskeleton organization & 5.58167E-07 & 1.1.12111E-05 & 2.710353929 \\
\hline GO:0007507 & Heart development & 1.51048E-05 & 0.0 .000231696 & 2.673988917 \\
\hline GO:0006950 & Response to stress & 8.30749E-09 & $2.42587 \mathrm{E}-07$ & 2.628016211 \\
\hline GO:0006979 & Response to oxidative stress & $2.57902 \mathrm{E}-05$ & 0.000364838 & 2.538312732 \\
\hline GO:0001558 & Regulation of cell growth & $1.62148 \mathrm{E}-06$ & 3.00709E-05 & 2.502837518 \\
\hline GO:0008283 & Cell proliferation & $6.44871 \mathrm{E}-15$ & $4.17534 \mathrm{E}-13$ & 2.426344333 \\
\hline $\mathrm{GO}: 0042981$ & Regulation of apoptosis & 5.36115E-05 & 0.000697636 & 2.304057989 \\
\hline $\mathrm{GO}: 0006917$ & Induction of apoptosis & 5.72349E-07 & 1.14703E-05 & 2.284267256 \\
\hline GO:0007155 & Cell adhesion & 1.1192E-19 & 1.1.24009E-17 & 2.229968617 \\
\hline GO:0055114 & Oxidation reduction & 6.80721E-05 & 0.000850389 & 1.515909961 \\
\hline
\end{tabular}

id, identification number; MAPKKK, mitogen- activated protein kinase kinase kinase; STZ, streptozotocin.

osmotic control. The experiments were repeated with at least three different cardiomyocyte cultures.

In the experiments, to inhibit p38 MAPK, ERK1/2, and JNK, cardiomyocytes were pretreated with or without 5 $\mu \mathrm{mol} / \mathrm{L}$ SB-203580, PD-98059 or SP-600125 for 30 minutes and then exposed to 5.5 or $25 \mathrm{mmol} / \mathrm{L}$ glucose for 48 hours.

\section{Cardiac Hypertrophy and Cardiomyocyte Cell Size Analysis}

At 8 weeks after diabetes was induced, the left ventricle was rinsed in PBS buffer and weighted to calculate the ratio of heart weight to body weight (HW/BW). Cardiac hypertrophy was analyzed by histology. Briefly, LV samples were fixed in $4 \%$ paraformaldehyde for 12 hours at room temperature and embedded in paraffin. After that, 5- $\mu \mathrm{m}$-thick sections were stained with hematoxylin and eosin (H\&E) and cardiomyocyte size was measured using NIH ImageJ software on micrographs. Approximately 100 randomly chosen cardiomyocytes from each group $(n=5)$ were analyzed to measure the cross-sectional cardiomyocyte area. ${ }^{18,19}$ Cardiomyocyte cell surface area was determined to assess cellular hypertrophy. ${ }^{16}$ Cells were visualized with a Nikon (Tokyo, Japan) microscope and images were captured at $40 \times$ magnification. At least 100 cells from randomly selected fields in one well were examined.

\section{Real-Time RT-PCR}

Total RNA was extracted from mouse LV tissues using the TRIzol reagent (Invitrogen) following the manufacturer's in-

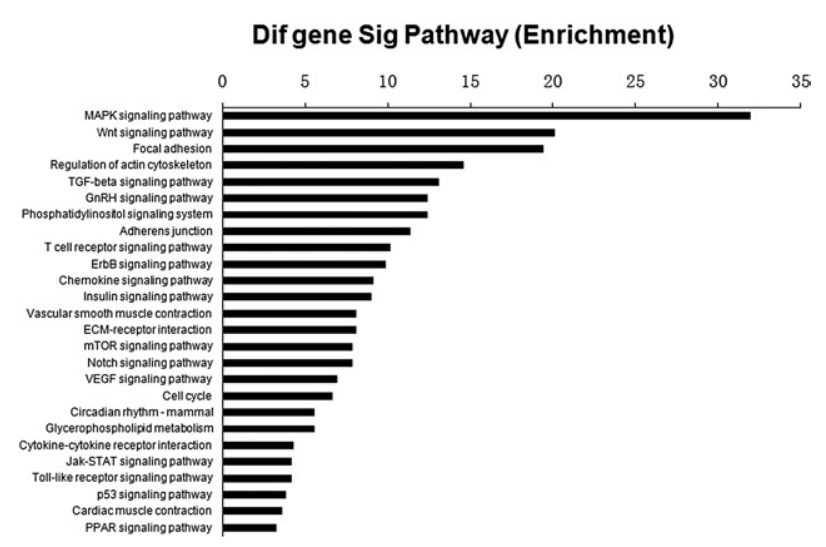

Figure 2. Pathway analysis based on miRNA-targeted genes, which showed significant pathways targeted by aberrantly expressed miRNAs. The vertical axis is the pathway category; the horizontal axis is the enrichment of pathways. 
Table 2. Significant Pathways That Are Enriched with Target Genes of MicroRNA

\begin{tabular}{|c|c|c|c|}
\hline Path_name & $P$ value & FDR & Target genes \\
\hline $\begin{array}{l}\text { MAPK signaling } \\
\text { pathway }\end{array}$ & 1.13E-32 & 5.557E-31 & $\begin{array}{l}\text { Pla2g4b, NF1, Dusp3, Dusp4, PLA2G4A, MAPK7, MAP2K1, NLK, GNG12, ELK4, } \\
\text { ACVR1B, RAPGEF2, MAPK8IP3, CACNB1, MAPK8IP1, NTF3, MAPK3, CRKL, } \\
\text { MRAS, DUSP16, TAOK2, NTRK2, MAP3K14, TAOK1, CaCng2, MAP3K7IP2, } \\
\text { FGF1, TAOK3, FASLG, DUSP5, RAF1, RPS6KA5, DUSP2, EVI1, MAP3K11, } \\
\text { FGF2, KRAS, RPS6KA1, PDGFA, MAP3K3, TGFB3, PPM1A, CACNG3, PPP3R1, } \\
\text { RASGRP4, MAP3K1, RASGRP1, PPP3CB, PPM1B, CACNA2D3, MEF2C, STK4, } \\
\text { CACNA1E, MAP3K7, CDC42, PAK1, JUND, MAP4K3, CACNA1C, Rasgrf1, Akt1, } \\
\text { TNFRSF1A, RAC1, MAP2K3, CHP, MAP3K5, HsPa1I, PPP3CA, MAP3K4, } \\
\text { TGFBR1, RAP1A, AKT3, Hspa2, BDNF, GNA12, RASA1, MAPK9, FOS, Sif, } \\
\text { RAP1B, MAP2K6, CRK, MAP2K4, FGFR3, NfatC2, FGF7, MAPK10, PDGFRA, } \\
\text { IL1R1, ACVR1C }\end{array}$ \\
\hline $\begin{array}{l}\text { Wnt signaling } \\
\text { pathway }\end{array}$ & 8.141E-21 & 2.002E-19 & $\begin{array}{l}\text { PPP2R1A, FBXW11, CTBP2, Csnk1e, Nkd2, NLK, VANGL1, BTRC, NFATC3, } \\
\text { Ctbp1, WNT7A, LEF1, CCND1, Crebbp, CSNK1A1, FZD7, SFRP1, SFRP2, } \\
\text { ROCK2, SMAD4, LRP6, PPP2R5C, PLCB1, Camk2b, SMAD3, PPP3R1, TBL1X, } \\
\text { DAAM1, NFAT5, PPP3CB, WNT1, CCND2, MAP3K7, SMAD2, Camk2a, } \\
\text { PPP2R5E, APC,SIAH1, RAC1, TCF7, CHP, PPP3CA, CSNK2A2, FZD9, MAPK9, } \\
\text { WNT3A, FOSL1, FZD10, Nfatc2, Ppp2ca, MAPK10, PRICKLE2, AXIN2 }\end{array}$ \\
\hline Focal adhesion & 3.979E-20 & 7.369E-19 & $\begin{array}{l}\text { MET, PDPK1, Ppp1CC, ZYX, COL3A1, MAP2K1, VEGFA, PXN, CHAD, ITGA11, } \\
\text { COL1A2, CRKL, COL1A1, CCND1, COL4A1, TNXB, VASP, MAPK3, RAF1, } \\
\text { ROCK2, PDGFA, Rasgrf1, COL11A2, PARVA, CAV2, PAK4, ITGB8, CCND2, } \\
\text { IGF1, ITGAV, IIK, PDGFC, PAK1, CDC42, ITGA2, RAC1, AKt1, COL6A3, RAP1A, } \\
\text { PPP1R12A, PIK3R1, COL5A3, ITGA3, COL5A2, FLT1, VAV1, AKT3, PTEN, } \\
\text { RAP1B, MAPK9, LAMC1, BCL2, COL2A1, FN1, CRK, RELN, MYLK, ITGA6, SLK, } \\
\text { MAPK10, PDGFRA }\end{array}$ \\
\hline $\begin{array}{l}\text { Regulation of actin } \\
\text { cytoskeleton }\end{array}$ & $2.714 \mathrm{E}-15$ & 3.086E-14 & $\begin{array}{l}\text { Pfn1, PDGFRA, GIT1, WASL, TMSL, CSK, Ppp1CC, GNG12, MYH9, PIP5K1A, } \\
\text { MRAS, PXN, ITGA11, LIMK1, FGF1, CRKL, FGF2, MAP2K1, ROCK2, Chrm2, } \\
\text { IQGAP2, PDGFA, ARHGEF12, RAF1, SSH2, MAPK3, PAK4, BAIAP2, KRAS, } \\
\text { ITGB8, TMSB4X, ITGAV, FN1, APC, PDGFC, PAK1, ENAH, MYH10, ITGA2, } \\
\text { CDC42, ARHGEF7, PPP1R12A, GNA12, ITGA3, RAC1, PIK3R1, Pip5K3, VAV1, } \\
\text { PFN2, CRK, MYLK, FGFR3, FGF7, CFL2, SLC9A1, ITGA6 }\end{array}$ \\
\hline $\begin{array}{l}\text { TGF-beta signaling } \\
\text { pathway }\end{array}$ & 8.813E-14 & $7.88 \mathrm{E}-13$ & $\begin{array}{l}\text { FST, ACVR1C, Nog, PPP2R1A, BMPR1A, ACVR1B, SMURF2, SMAD7, MAPK3, } \\
\text { Crebbp, SMAD5, SMURF1, E2F5, ROCK2, SMAD4, RBL1, TGFB3, INHBB, } \\
\text { SMAD3, LEFTY1, ACVR2A, SMAD2, ACVR2B, GDF6, PITX2, SP1, TGFBR1, } \\
\text { Acvrl1, ACVR1, Id1, Ppp2ca, CHRD }\end{array}$ \\
\hline $\begin{array}{l}\text { GnRH signaling } \\
\text { pathway }\end{array}$ & 4.029E-13 & $2.91 \mathrm{E}-12$ & $\begin{array}{l}\text { CALM3, MMP14, MAPK10, Pla2g4b, MAPK7, PLA2G4A, CALM2, ITPR3, MAP2K1, } \\
\text { CALM1, ITPR1, MAP3K3, Adcy2, PLCB1, MAP3K1, Camk2b, RAF1, MAPK3, } \\
\text { ADCY9, Adcy3, ADCY1, KRAS, Camk2a, CACNA1C, MAP2K3, MAP3K4, Ptk2b, } \\
\text { CDC42, Gna11, MAP2K6, MAP2K4, GNAQ, HBEGF, MAPK9 }\end{array}$ \\
\hline $\begin{array}{l}\text { Phosphatidylinositol } \\
\text { signaling system }\end{array}$ & 4.489E-13 & 3.153E-12 & $\begin{array}{l}\text { PIK3C2A, CALM2,ITPR3, PIP5K1A, OCRL, CALM1, ITPR1, ImPa2, PLCB1, PI4KA, } \\
\text { PI4KB, IPPK, CDS2, DGKB, INPP5F, ITPKB, DGKD, PIK3R1, INPP5B, PIB5PA, } \\
\text { DGKG, INPP5A, PLCG1, PTEN, ITPK1, Pip5k3, DGKH, CALM3, SYNJ1 }\end{array}$ \\
\hline Adherens junction & 4.639E-12 & $2.535 \mathrm{E}-11$ & $\begin{array}{l}\text { ACVR1C, WASL, MET, NLK, ACVR1B, CTNND1, PTPN1, LEF1, PTPRF, CTNNA1, } \\
\text { Crebbp, MAPK3, SMAD4, Snai1, SMAD3, BAIAP2, WASF3, MAP3K7, SMAD2, } \\
\text { PVRL2, CDC42, TCF7, RAC1, CSNK2A2, TGFBR1, SSX2IP, YES1, SLK }\end{array}$ \\
\hline $\begin{array}{l}\text { T cell receptor } \\
\text { signaling pathway }\end{array}$ & $7.094 \mathrm{E}-11$ & $3.668 \mathrm{E}-10$ & $\begin{array}{l}\text { SLK, NCK2, MAP2K1, CD4, NFATC3, MAP3K14, CBLB, CD28, CBL, RAF1, } \\
\text { MAPK3, RASGRP1, KRAS, PAK4, PPP3R1, NFAT5, PPP3CB, MAP3K7, PAK1, } \\
\text { CDC42, AKt1, CHP, PPP3CA, PIK3R1, PLCG1, VAV1, FOS, CSf2, AKT3, MAPK9, } \\
\text { DLG1, Nfatc2 }\end{array}$ \\
\hline $\begin{array}{l}\text { ErbB signaling } \\
\text { pathway }\end{array}$ & $1.344 \mathrm{E}-10$ & $6.612 \mathrm{E}-10$ & $\begin{array}{l}\text { NRG2, CDKN1B, GAB1, MAP2K1, NCK2, MAPK3, ABL1, CRKL, CBLB, RAF1, } \\
\text { CBL, ABL2, KRAS, FRAP1, Camk2b, ERBB4, PAK4, Camk2a, PAK1, Akt1, } \\
\text { PIK3R1, AKT3, PLCG1, MAPK9, CRK, MAP2K4, HBEGF, MAPK10 }\end{array}$ \\
\hline $\begin{array}{l}\text { Chemokine signaling } \\
\text { pathway }\end{array}$ & $8.412 \mathrm{E}-10$ & 3.575E-09 & $\begin{array}{l}\text { GNB5, WASL, CSK, CCl3, MAP2K1, GNG12, Gip, PXN, STAT3, CX3CR1, MAPK3, } \\
\text { ADRBK2, GNG2, CRKL, GRK6, CX3CL1, ROCK2, RAF1, PREX1, PLCB1, KRAS, } \\
\text { GNAI3, Adcy2, Stat1, GNAI2, ADCY9, AdCy3, ADCY1, CXCL11, CDC42, PAK1, } \\
\text { CCL1, RAC1, Akt1, CCL5, PIK3R1, Ptk2b, RAP1A, AKT3, VAV1, RAP1B, CRK, } \\
\text { GNB2 }\end{array}$ \\
\hline $\begin{array}{l}\text { Insulin signaling } \\
\text { pathway }\end{array}$ & 1.018E-09 & 4.185E-09 & $\begin{array}{l}\text { CALM3, MAPK10, PDPK1, SLC2A4, PpP1CC, HK3, CALM2, PTPN1, EIF4E2, } \\
\text { PRKAR2A, PTPRF, CRKL, MAP2K1, CBLB, CALM1, CBL, PPP1R3A, FRAP1, } \\
\text { FLOT2, RHEB, PPP1R3D, RAF1, HK1, MAPK3, PPP1R3B, KRAS, TRIP10, Akt1, } \\
\text { FOXO1, PIK3R1, PPARGC1A, EIF4E, MAPK9, CRK, AKT3 }\end{array}$ \\
\hline $\begin{array}{l}\text { Vascular smooth } \\
\text { muscle } \\
\text { contraction }\end{array}$ & 8.36E-09 & 2.757E-08 & $\begin{array}{l}\text { Pla2g4b, Ppp1cC, PLA2G4A, CALM2, MAP2K1, ITPR3, MAPK3, ROCK2, CALM1, } \\
\text { RAF1, ITPR1, PLCB1, CALD1, PRKCE, AdCy2, ARHGEF12, ARHGEF11, ADCY9, } \\
\text { Adcy3, ADCY1, KCNMA1, CACNA1C, PPP1R12B, PPP1R12A, GNA12, Gna11, } \\
\text { PRKG1, GNAQ, MYLK, CALM3 }\end{array}$ \\
\hline $\begin{array}{l}\text { ECM-receptor } \\
\text { interaction }\end{array}$ & 8.649E-09 & 2.836E-08 & $\begin{array}{l}\text { HSPG2, COL3A1, CHAD,ITGA11,COL1A2, COL1A1, COL4A1, TNXB, SDC4, SDC2, } \\
\text { SDC1, COL11A2, CD44, ITGB8, ITGAV, ITGA2, COL6A3, COL5A3, ITGA3, } \\
\text { COL5A2, LAMC1, COL2A1, FN1, RELN, ITGA6 }\end{array}$ \\
\hline
\end{tabular}


Table 2. Continued

\begin{tabular}{|c|c|c|c|}
\hline Path_name & $P$ value & FDR & Target genes \\
\hline $\begin{array}{l}\text { mTOR signaling } \\
\text { pathway }\end{array}$ & 1.405E-08 & 4.343E-08 & $\begin{array}{l}\text { PDPK1, ULK1, CAB39, VEGFA, MAPK3, EIF4E2, RICTOR, STK11, RPS6KA1, } \\
\text { FRAP1, RHEB, DDIT4, IGF1, AKt1, PIK3R1, AKT3, HIF1A, EIF4B, EIF4E }\end{array}$ \\
\hline $\begin{array}{l}\text { Notch signaling } \\
\text { pathway }\end{array}$ & $1.46 \mathrm{E}-08$ & $4.488 \mathrm{E}-08$ & $\begin{array}{l}\text { CTBP2, Jag2, NOTCH3, NOTCH1, JAG1, Ctbp1, Crebbp, NUMB, Ncor2, DLL1, } \\
\text { DLL4, Hes5, KAT2B, HN1, MAML3, MAML1, RBPJ, Psen2 }\end{array}$ \\
\hline $\begin{array}{l}\text { VEGF signaling } \\
\text { pathway }\end{array}$ & $1.258 \mathrm{E}-07$ & 3.496E-07 & $\begin{array}{l}\text { Pla2g4b, PTGS2, PLA2G4A, MAP2K1, VEGFA, PXN, NFATC3, MAPK3, RAF1, } \\
\text { KRAS, PPP3R1, NFAT5, PPP3CB, CDC42, RAC1, Akt1, CHP, PIK3R1, PPP3CA, } \\
\text { AKT3, PLCG1, Nfatc2 }\end{array}$ \\
\hline Cell cycle & 2.445E-07 & $6.165 \mathrm{E}-07$ & $\begin{array}{l}\text { CCNE1, CDC25A, CDKN1B, CDC23, YWHAB, E2F3, YWHAE, CCND1, YWHAG, } \\
\text { ABL1, Crebbp, YWHAH, MYT1, YWHAQ, SMAD4, CDC14A, RBL1, CDK6, } \\
\text { TGFB3, SMAD3, WEE1, CCND2, SMAD2, CCnb2, CDKN1C, YWHAZ, RB1, } \\
\text { CHEK1 }\end{array}$ \\
\hline $\begin{array}{l}\text { Circadian rhythm, } \\
\text { mammal }\end{array}$ & 2.809E-06 & $6.092 \mathrm{E}-06$ & CLOCK, CRY2, Csnk1e, NR1D1, BHLHE41, PER2, PER1, ARNTL \\
\hline $\begin{array}{l}\text { Glycerophospholipid } \\
\text { metabolism }\end{array}$ & 2.906E-06 & $6.276 \mathrm{E}-06$ & $\begin{array}{l}\text { CHKA, GPD1L, PISD, CDS2, AGPAT3, AGPAT1, DGKB, ETNK1, GPD2, DGKD, } \\
\text { DGKG, LYPLA2, CHPT1, Ache, PHOSPHO1, DGKH, Pgs1, Pla2g4b, PLA2G4A }\end{array}$ \\
\hline $\begin{array}{l}\text { Cytokine-cytokine } \\
\text { receptor } \\
\text { interaction }\end{array}$ & $5.155 \mathrm{E}-05$ & 9.309E-05 & $\begin{array}{l}\text { BMPR1A, Cd40, LEPR, CCl3, LIFR, Tnfrsf25, VEGFA, ACVR1B, PRLR, LTB, } \\
\text { CX3CR1, CX3CL1, FASLG, LIF, IL13RA1, GHR, TNFRSF21, PDGFA,TGFB3, } \\
\text { KITLG, INHBB, CNTFR, CXCL11, ACVR2A, ACVR2B, CCL1, PDGFC, TNFRSF1A, } \\
\text { CCL5, NGFR, CNTF, TGFBR1, TNFRSF19, ACVr11, FLT1, CSf2, ACVR1, EDA, } \\
\text { IL1R1, PDGFRA, MET }\end{array}$ \\
\hline $\begin{array}{l}\text { Jak-STAT signaling } \\
\text { pathway }\end{array}$ & $6.844 \mathrm{E}-05$ & 0.0001202 & $\begin{array}{l}\text { PIAS3, STAM, LEPR, LIFR, PRLR, STAT3, CCND1, PIAS1, LIF, Crebbp, IL13RA1, } \\
\text { CBLB, CBL, GHR, Stat1, CNTFR, CCND2, CNTF, Akt1, PIK3R1, CSf2, PIM1, } \\
\text { AKT3, SPRY4, SPRED2, SPRY2, SPRED1, SPRY1 }\end{array}$ \\
\hline $\begin{array}{l}\text { Toll-like receptor } \\
\text { signaling pathway }\end{array}$ & 7.645E-05 & 0.0001326 & $\begin{array}{l}\text { Cd40, CCl3, IRAK1, MAP2K1, MAP3K7IP2, MAPK3, Stat1, CXCL11, MAP3K7, } \\
\text { MAP2K3, CCL5, RAC1, AKt1, PIK3R1, FOS, MAP2K6, AKT3, MAPK9, MAP2K4, } \\
\text { TIRAP, MAPK10 }\end{array}$ \\
\hline $\begin{array}{l}\text { p53 signaling } \\
\text { pathway }\end{array}$ & 0.0001592 & 0.0002601 & $\begin{array}{l}\text { BAI1, CCNE1, CCND1, IGFBP3, SESN1, PPM1D, CDK6, PMAIP1, CCND2, } \\
\text { RCHY1, IGF1, CCnb2, SIAH1, Lrdd, PTEN, CHEK1 }\end{array}$ \\
\hline $\begin{array}{l}\text { Cardiac muscle } \\
\text { contraction }\end{array}$ & 0.0002602 & 0.0004089 & $\begin{array}{l}\text { UQCRB, Atp1a3, CACNB1, ATP2A2, Uqcrc2, Cacng2, CACNG3, Cox6a1, } \\
\text { CACNA2D3, Tnnc1, CACNA1C, TPM3, SLC8A1, SLC9A6, ATP1A1, SLC9A1, } \\
\text { Myl4 }\end{array}$ \\
\hline $\begin{array}{l}\text { PPAR signaling } \\
\text { pathway }\end{array}$ & 0.0005643 & 0.0008409 & $\begin{array}{l}\text { PDPK1, ACOX1, SCD, APOA5, Angptl4, PPARA, ACSL1, Ilk, ANGPTL2, Cyp27a1, } \\
\text { LPL, SCD5, ACSL4, NR1H3, Rxra }\end{array}$ \\
\hline
\end{tabular}

ECM, extracellular matrix; MAPK, mitogen-activated protein kinase; PPAR, peroxisome proliferator-activated receptor; TGF, transforming growth factor; VEGF, vascular endothelial growth factor.

structions. Only the highly pure RNAs (ratio of 260/280 > 1.8 and 260/230 > 1.8) were used for downstream assay. Real-time RT-PCR for atrial natriuretic peptide (ANP), brain natriuretic peptide (BNP), $\beta$-myosin heavy chain $(\beta-\mathrm{MHC})$, and glyceraldehyde-3-phosphate dehydrogenase (GAPDH) were performed using the primers as follows: ANP (GenBank number: NM_008725.2) forward, 5'- TGACAGGATTGGAGCCCAGAG-3'; reverse, 5'- AGCTGCGTGACACACCACAAG-3'; BNP (GenBank number: NM_008726.4) forward, 5'-ATCGGATCCGTCAGTCGTTTG-3'; reverse, 5'CCAGGCAGAGTCAGAAACTGGAG-3'; $\beta$-MHC (GenBank number: NM_080728.2) forward, 5'-TAACCCGAGGCAAGCTCACA-3'; reverse, 5'-CACAATCATGCCGTGCTGAC-3'; GAPDH (GenBank number: NM_008084.2) forward, 5'- TGTGTCCGTCGTGGATCTGA-3'; reverse, 5'TTGCTGTTGAAGTCGCAGGAG-3'. The neonatal rat cardiomyocytes were cultured in DMEM. miR-373 levels were detected by real-time quantitative PCR. The sequences of primers, which were designed for miR-373 and U6 (for normalization), to analyze their expression, were as follows: miR-373 stem-loop primer 5'-GTCGTATCCAGTGCAGGGTCCGAGGTATTCGCACTGGATACGACACACC3'; miR-373 forward, 5'-GTGCTTCGATTTTGGGG-3'; miR373 reverse, 5'-GTGCAGGGTCCGAGGT-3'; U6 forward, 5'-CTCGCTTCGGCAGCACA-3'; and U6 reverse transcript and reverse, 5'-AACGCTTCACGAATTTGCGT-3'. Relative gene expression levels were calculated by the $2^{-\Delta \Delta C T}$ method. ${ }^{20}$

\section{Microarray Analysis and Target Prediction}

miRNAs were purified from total RNA by using the mirVana miRNA Isolation Kit (Ambion, Austin, TX). Purification of miRNA from three cardiac tissues in each group was labeled and used for miRNA expression analysis by using the CapitalBio Mammalian miRNA Array V4.0 (CapitalBio Corp.) containing 1320 probes including human, mouse, and rat mature miRNAs. Differentially regulated miRNAs were defined as those with either _0.5- or 1.5 -fold changes in expression for both arrays from diabetic or nondiabetic mouse heart. The normalized microarray data were validated by using real-time PCR. The commonly used and accurate Sanger miRNAs database (http://microrna.sanger.ac.uk) was used to predict potential miRNA targets. The top $25 \%$ of miRNA targets that had been assigned the highest numbers of miRNAs interaction sites were collected.

\section{GO Category and Pathway Analysis}

The top $25 \%$ of miRNA targets were subjected to GO term analysis, which was applied to organize genes into 


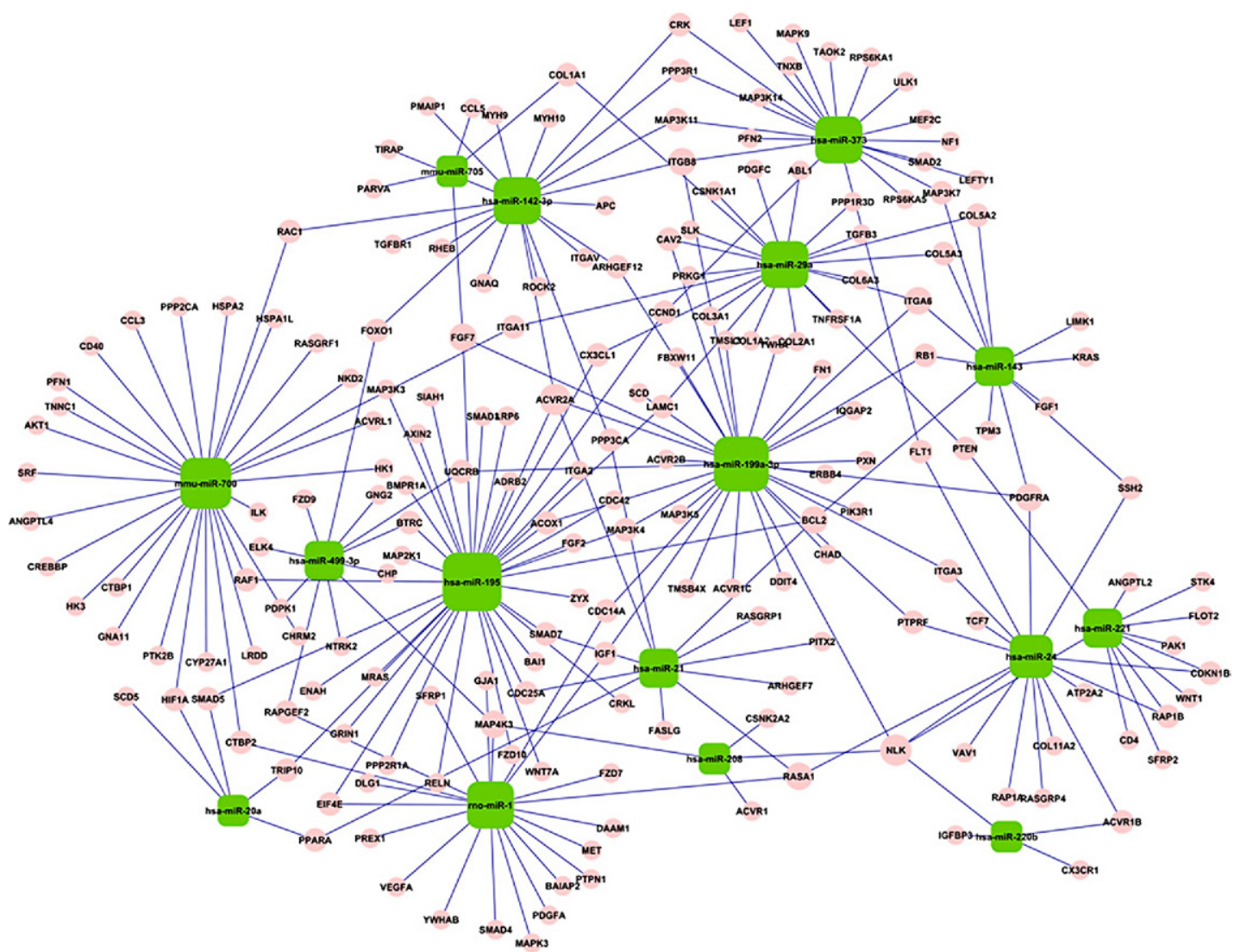

Figure 3. MicroRNA-gene network. Square represents miRNAs; circle represents target genes; straight line represents miRNA-gene relationship. The size of the square represents the degrees of miRNAs; larger degrees have larger numbers of target genes.

hierarchical categories on the basis of biological process, molecular function, and subcellular localization. ${ }^{21}$ Generally, a two-sided Fisher's exact test and $\chi^{2}$ test were used to classify the GO category, and the false discovery rate (FDR) was calculated to correct the $P$ value. We computed $P$ values for all of the different genes in all GO biological categories; the threshold of significance was defined as $P<0.01$ and FDR $<0.05$. Enrichment provides a measure of the significance of the function: As the enrichment increases, the corresponding function is more significant. Within the significant category, the enrichment Re was given by the following: Re $(n / n) /(N / N)$, where $n_{f}$ is the number of differential genes within the particular category, $n$ is the total number of genes within the same category, $N_{f}$ is the number of differential genes in the entire microarray, and $N$ is the total number of genes in the microarray.

Meanwhile, the top 25\% of miRNA targets were collected, and subjected to KEGG pathway annotation. Similarly, pathway analysis was used to determine the significant pathway of the differential genes. Fisher's exact test and $\chi^{2}$ test were used to select $t$ significant pathway, and the threshold of significance was defined as $P<0.01$ and FDR $<0.05$. The enrichment Re was calculated like the equation above. Furthermore, the network of miRNA-mRNA interaction, representing the critical miRNAs and their targets, was established based on the integration of GO function and KEGG analysis findings.

\section{miRNA Mimic Transfection}

The primary neonatal rat cardiomyocytes were seeded in antibiotic-free medium for 24 hours before transfection. Lipofectamine 2000 (Invitrogen) was used to transfect cells with miR-373 $(20 \mathrm{nmol} / \mathrm{L})$ mimic (Dharmacon RNA Technologies) for 48 hours according to the manufacturer's instructions. miRIDIAN miRNA mimic negative controls (Dharmacon RNA Technologies) were used for control transfections. Cells were collected after 48 hours of miRNA mimic transfections for quantification of miRNA and protein expression.

\section{Western Blot Analysis Significant Pathways}

Total proteins isolated from LV tissues or cultured cardiomyocytes were prepared by standard procedures and quantified by the bicinchoninic acid (BCA) protein assay (Pierce, USA). A total of $30 \mu \mathrm{g}$ protein in each sample was subjected to $10 \%$ SDS-PAGE gel by electrotransfer onto polyvinylidene difluoride membranes (Millipore, USA). The expression or phosphorylation of p38, ERK1/2, and JNK were determined by using specific antibodies against total-p38, -ERK1/2, -JNK and phospho-p38, 


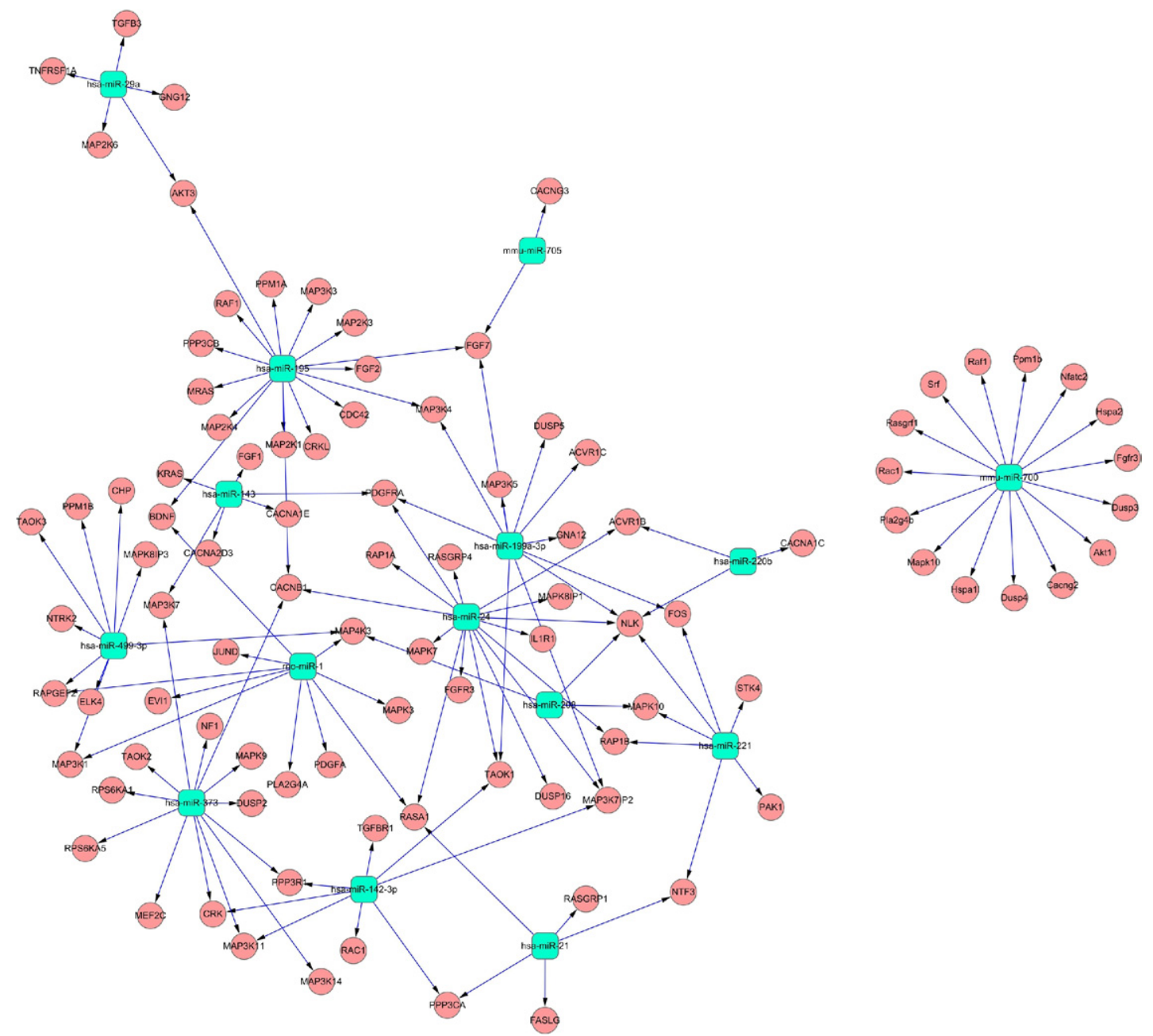

Figure 4. The interaction between MAPK signaling pathway-related genes and miRNAs. Square represents miRNAs; circle represents target genes; straight line represents miRNAs-MAPK signaling pathway-related gene relationship.

-ERK1/2, and -JNK, respectively. The primary antibody against MEF2C was used to analyze MEF2C protein expression. After scanning, results were quantified by the $\mathrm{NIH}$ ImageJ program.

\section{Statistical Analysis}

All experimental data are expressed as mean \pm SD and were analyzed by Wilcoxon Mann-Whitney method and Kruskal-Wallis test. A $P$ value of 0.05 or less was considered significant.

\section{Results}

\section{Hyperglycemia Induces Cardiac Hypertrophy and Neonatal Rat Cardiomyocyte Hypertrophy}

At 72 hours after STZ injection, blood glucose was elevated to $24.0 \pm 3.0 \mathrm{mmol} / \mathrm{L}$ in diabetic mice versus $8.3 \pm$ $0.6 \mathrm{mmol} / \mathrm{L}$ in the C57BL6 controls. Throughout the 8-week study period, mice with STZ-induced diabetes displayed severe hyperglycemia. At the end of 8 weeks after diabetes was induced, increased LVHW/BW ratios were observed in diabetic mice (Figure 1A). At the same time, histological analysis showed that the cross-sectional area of cardiomyocytes increased in the diabetic heart (Figure 1, B and C). Furthermore, ANP, BNP, and $\beta-\mathrm{MHC}$, markers of cardiac hypertrophy, were upregulated in diabetic hearts as assessed by real-time RT-PCR (Figure 1D). Further in vitro investigation showed that high glucose increased the size of cultured cardiomyocytes exposed to $25 \mathrm{mmol} / \mathrm{L}$ glucose for 48 hours (Figure 1E).

\section{Altered Expression of miRNAs and Their Target Genes in the Diabetic Heart}

Using miRNA microarray analysis, we evaluated miRNA expression profiles of three diabetic heart samples and three nondiabetic heart samples (fold change: _0.5- or 1.5; false discovery rate: $\leq 0.05$ ). We identified 19 miRNAs that were deregulated greater than twofold, and 16 were further verified by real-time PCR. Overall, miR-195, miR-199a-3p, miR-700, miR-142-3p, miR-24, miR-21, miR-221, miR-499$3 p$, miR-208a, and miR-705 have often been found to be 

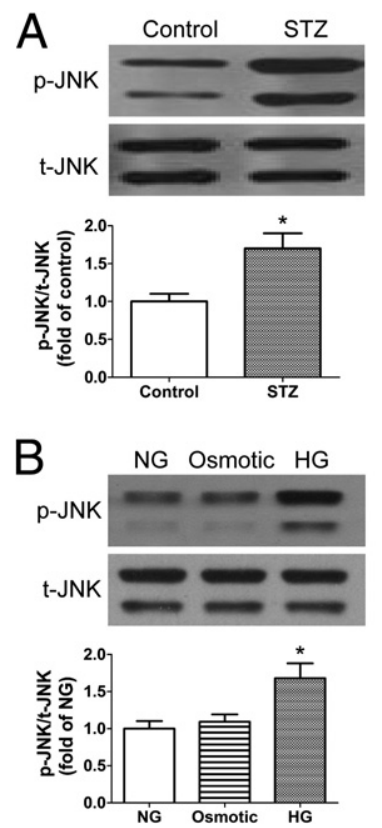
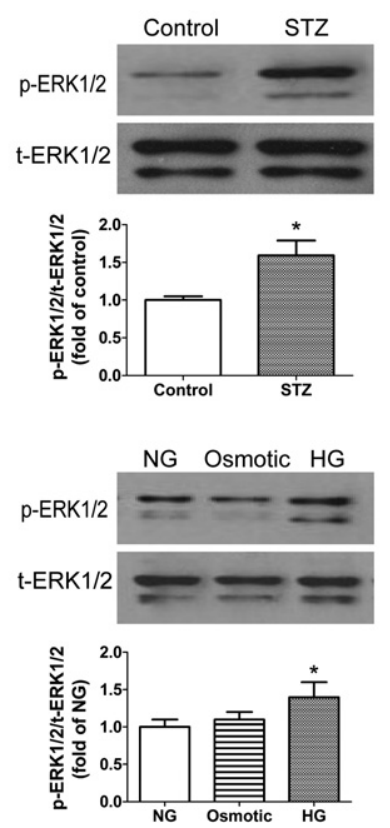
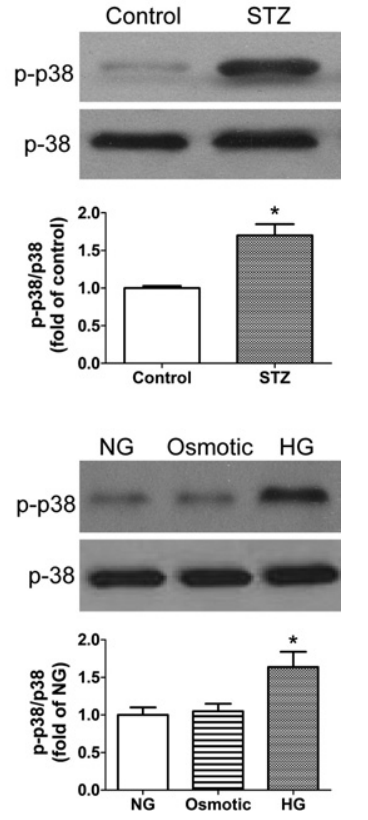

Figure 5. Activation of JNK, ERK $1 / 2$, and $p 38$ MAPK in cardiomyocyte hypertrophy induced by hyperglycemia. A: Western blot analysis of JNK, ERK1/2, and p38 MAPK protein levels in cardiac tissue of diabetic mice (upper panels) Results are shown for 3 mice in each group. B: Western blot analysis of ERK1/2, JNK, and P38 MAPK in neonatal rat cardiomyocytes (upper panels). Data are mean \pm SD from at least three different cell cultures. For $\mathbf{A}$ and $\mathbf{B}$, bar graph (lower panels) shows expression ratio of phospho-JNK over total JNK, phospho-ERK over total ERK, and phospho-p38 over total p38, respectively. ${ }^{*} P<0.05$ compared with control or NG. upregulated, whereas miR-29, miR-1, miR-373, miR-143, miR-20a, and miR-220b have been found to be down-regulated in STZ-induced diabetes when compared with controls. By using the Sanger database, 3212 target genes of the 16 changed miRNAs were collected.

\section{GO Function and KEGG Pathway Enrichments}

To gain a better understanding of the functional roles of the predicted miRNAs target genes and related regulatory network, we looked for target enrichment in $\mathrm{GO}$ and KEGG. On the basis of the top 25\% miRNA targets, 31 GO terms were found to be involved in the diabetic cardiomyopathy (Table 1). The five high-enrichment GOs were insulin-like growth factor receptor signaling, JNK cascade, leukocyte adhesive activation, transmembrane receptor protein serine/threonine kinase signaling, MAPKKK cascade, and collagen fibril organization. They were involved in proliferative, metabolic, extracellular matrix, adhesive, apoptosis, signal transduction, and heart physiology. KEGG pathway analysis of the top $25 \%$ of the predicted targets that were assigned the highest numbers of miRNA target sites revealed 26 KEGG pathways enriched in diabetic cardiomyopathy (Figure 2). Many of these signaling pathways, such as MAPK, Wnt, TGF- $\beta$, VEGF, JAK/STAT, and mTOR have been shown to participate in the development of cardiac hypertrophy (Table 2). Among the list of high-enrichment KEGG pathways of these miRNA-targeted genes, MAPK signaling pathway was significantly prominent. A similar phenomenon was also observed in $\mathrm{GO}$ analysis.

In addition to providing the interactions of miRNAs and target genes, the common targets of $\mathrm{GO}$ analysis and KEGG pathway were filtered out, miRNAs-gene network analysis integrated changed miRNAs, and 192 critical mRNAs were further generated (Figure 3). Intriguingly, the most overrepresented miRNA targets belonged to the
MAPK signaling pathway (Figure 4), which is known to be involved in the hypertrophic growth in response to overpressure by affecting the activities of the three bestcharacterized MAPKs including extracellular signal-regulated kinase (ERK1/2), c-Jun NH2-terminal kinase (JNK), and p38 mitogen-activated protein kinase (p38). These pathway analyses illustrate some of the possible roles of the highly expressed miRNAs in cardiac hypertrophy of diabetic mice.

\section{Experimental Verification the MAPK Signaling Pathway}

To further investigate whether MAPK signaling pathway mediates cardiomyocyte hypertrophy induced by hyperglycemia, we investigated the role of JNK, ERK1/2, and p38 MAPK in the heart in diabetes and in the cardiomyocytes exposed high glucose. Our results showed that diabetes in the heart causes significant upregulation of JNK, ERK1/2, and p38 (Figure 5A) in association with morphometrically demonstrable cardiomyocyte hypertrophy and ANP, BNP, and $\beta-\mathrm{MHC}$ upregulation (Figure 1). Exposure of cardiomyocytes to $25 \mathrm{mmol} / \mathrm{L}$ glucose for 48 hours showed similar upregulation of JNK, ERK $1 / 2$, and p38 MAPK protein levels compared with $5.5 \mathrm{mmol} / \mathrm{L}$ glucose (NG) and $25 \mathrm{mmol} / \mathrm{L}$ L-glucose (Figure 5B).

\section{miR-373 Mediates Glucose-Induced Cardiomyocyte Hypertrophy via Target Gene MEF2C}

To gain insight into the possible functional roles of miRNAs in diabetic cardiomyopathy, miR-373 levels in neonatal rat cardiomyocytes were modulated by transfecting the cells with miRIDIAN miR-373 mimic. A negative control miRNAs mimic was used as control. Trans- 
A

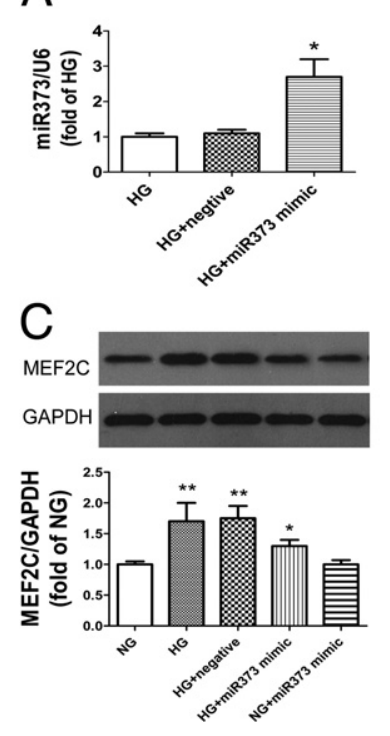

B

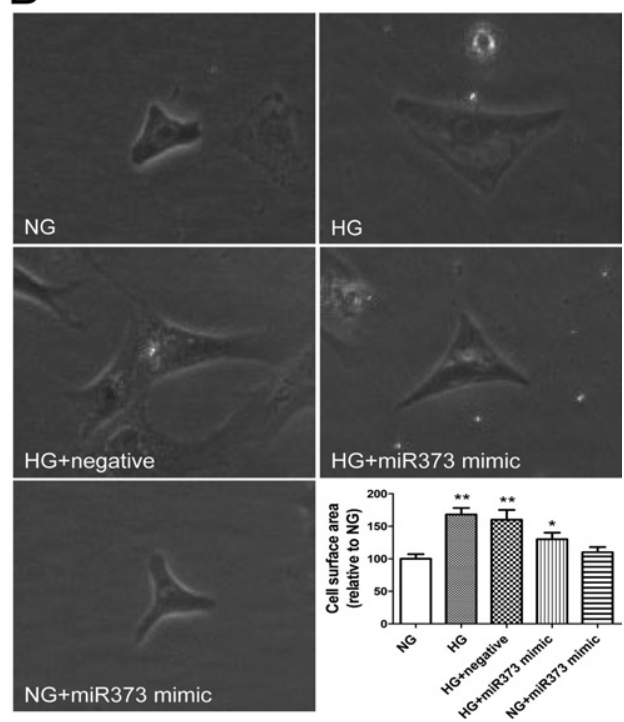

Figure 6. Neonatal rat cardiomyocytes were exposed to high glucose and transfected with miR-373 mimic. A: miR373 expression levels in cardiomyocytes 48 hours posttransfection were determined using real-time PCR. miRNA mimic negative controls were used for control transfections. ${ }^{*} P<$ 0.05 compared with control-transfected cardiomyocytes. B: Overexpression of miR-373 decreased cell surface area as compared with HG. C: Western blot analysis of MEF2C in miR-373-overexpressing cardiomyocytes (upper panels) Quantitative analysis MEF2C protein levels by densitometry (lower panels). ${ }^{*} P<0.05$ compared with $\mathrm{HG} ;{ }^{* * *} P<0.05$ compared with NG. fection with miR-373 mimic in glucose-exposed cells significantly increased the expression of miR-373 and decreased the cell size as measured by cell surface area (Figure 6, A and B). We also observed that the protein level of MEF2C was reduced when overexpressing miR373 (Figure 6C). The results suggested that MEF2C was a target gene of miR-373.

\section{miR-373 Down-Regulation Is Dependent on p38 MAPK Pathway}

To understand the relationship between miR-373 and MAPK signaling pathway in cardiac myocytes, activation of p38 MAPK, ERK1/2, and JNK was evaluated by Western blot. As shown in Figure 7, high glucose significantly increased the phosphorylation levels of p38 MAPK, ERK1/2, and JNK, but miR-373 mimic had no effect on phospho-p38 MAPK, phospho-ERK1/2, and phosphoJNK expression. To further explore the functional role of p38 MAPK, ERK1/2, and JNK, we used SB-203580, PD98059, and SP-600125 to test whether inhibition of p38 MAPK, ERK 1/2, and JNK could influence the effect of miR-373 on cardiac hypertrophy and MEF2C expression. Of note, a p38 MAP kinase inhibitor SB203580 significantly decreased miR-373 levels and inhibited high-glu- cose-induced hypertrophy (Figure 8, A-C), whereas PD98059 or SP600125 had no effect on miR-373 levels and cell size (data not shown). Furthermore, administration of SB-203580 led to a reduction in MEF2C levels compared with those in the HG group (Figure 8D).

\section{Discussion}

It is well demonstrated that miRNAs levels are altered in response to hypertrophic stimulation. 9,22,23 Therefore, it is reasonable to expect that dysregulation of miRNAs may related to diabetes-induced cardiac hypertrophy. With the use of miRNA microarray hybridization, we found 16 miRNAs that were expressed in diabetic mouse heart tissues. In them, 10 miRNAs, including miR-195, miR199a-3p, miR-700, miR-142-3p, miR-24, miR-21, miR221, miR-499-3p, miR-208a, and miR-705 were upregulated, and six miRNAs, including miR-29, miR-1, miR-373, miR-143, miR-20a, and miR-220b were down-regulated. In the late stage of diabetes, cardiac hypertrophy and subsequent heart failure are prominent features of diabetic cardiomyopathy. ${ }^{12,24}$ Consistent with previous reports, we have noted thatboth the HW/BW ratio and the cross-sectional surface area of cardiomyocytes were significantly increased in diabetic mice, and that ANP, BNP,
A
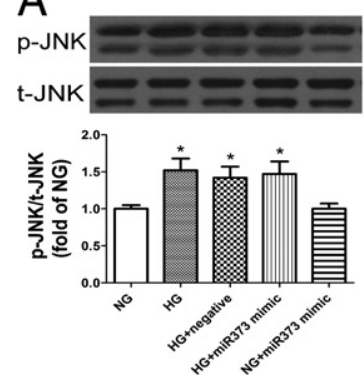

B
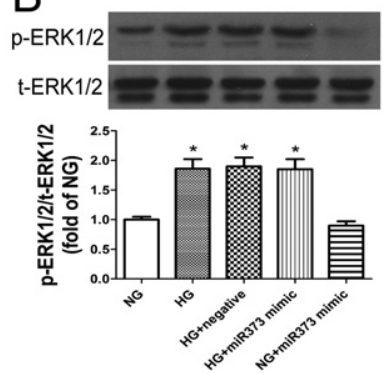

C

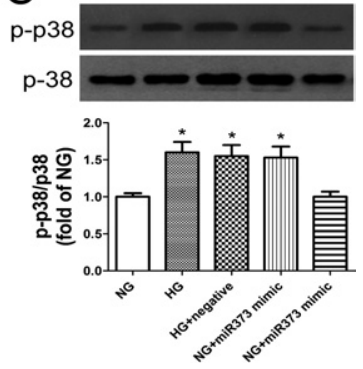

Figure 7. Effect of miR-373 mimic on expression of JNK, ERK, and p38 MAPK. Representative Western blot of phospho-JNK and total JNK, phospho-ERK1/2 and total ERK1/2, and phospho-p38 and total p38 in myocytes after miR-373 mimic transfected (upper panels, A-C). For A-C, bar graph (lower panels) shows expression ratio of phospho-JNK over total JNK, phospho-ERK1/2 over total ERK1/2, and phosphop38 over total p38, respectively. ${ }^{*} P<0.05$ compared with control or NG. 
A

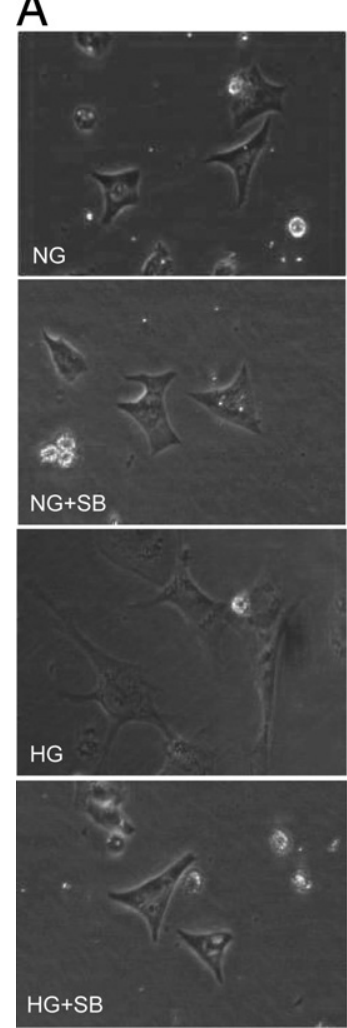

B
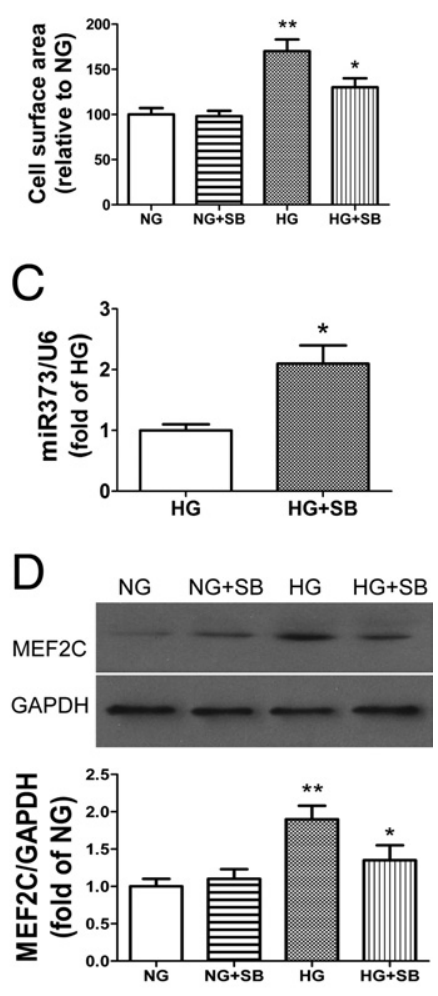

Figure 8. The effect of p38 inhibitor on cardiac myocyte hypertrophy, miR-373, and MEF2C expression. A: Cardiac myocyte size treated with or without p38 inhibitor in the presence or absence of high glucose. B: Quantitative analysis of cardiomyocyte surface area. C: Effect of p38 inhibitor on miR-373 expression levels in cardiomyocytes. D: Representative Western blot of $\mathrm{MEF} 2 \mathrm{C}$ protein expression levels (upper panels). Quantitative analysis MEF2C protein levels by densitometry (lower panels). ${ }^{*} P<0.05$ compared with $\mathrm{HG} ;{ }^{* * *} P<0.05$ compared with $\mathrm{NG}$.

and $\beta$-MHC expressed high levels in the diabetic heart. These results suggest that miRNAs have an important role in cardiac hypertrophy in STZ-induced diabetes.

To further investigate the functional significance of these miRNAs and related regulatory networks in diabetic cardiomyopathy, heuristic information of the miRNA target genes was needed. By using the Sanger database, 3212 target genes of the 16 miRNAs were collected. These 3212 target genes were then carefully annotated by the GO function and KEGG pathway analysis. GO is one of the most important tools for the representation and processing of information about gene products and functions. ${ }^{21,25}$ KEGG, a database of biological systems that integrates genomic, chemical, and systemic functional information, was used to find out the significant pathways of the different genes. ${ }^{26-28}$ For 16 changed miRNAs in the present study, $31 \mathrm{GO}$ functions and 26 KEGG pathways were annotated. GO analysis showed that insulinlike growth factor receptor signaling, JNK cascade, leukocyte adhesive activation, transmembrane receptor protein serine/threonine kinase signaling, MAPKKK cascade, and collagen fibril organization were abundant among the significantly enriched ones. This functional identity revealed by GO confirmed that changed miRNAs preferentially target genes involved in a wide spectrum of

regulatory functions and selected biological processes. KEGG further provides a mapping between genes and pathways. The signaling pathways enriched in diabetic cardiomyopathy are those associated with proliferative, survival, apoptotic, adhesive, and metabolic functions. Some of them have already been reported to take part in cardiac hypertrophy. For example, MAPK pathways are activated in cardiac tissue of TAC-induced hypertrophy mouse model. ${ }^{29,30}$ Activation of Wnt signaling is critical in the chronic phase of pressure overload cardiac hypertrophy in rats. ${ }^{31}$ TGF- $\beta 1$ may play a role in cardiac hypertrophy by inducing expression of $\beta$-MHC and skeletal $\alpha$-actin in cardiomyocytes. ${ }^{32}$ Upregulated VEGF signaling appears to contribute, at least in part, to endothelin1 -induced neonatal rat cardiomyocyte hypertrophy. ${ }^{33}$ The JAK/STAT pathway was found to be activated in rat hearts with pressure overload-induced hypertrophy. ${ }^{34,35}$ A specific inhibitor of mTOR, rapamycin, attenuates the hypertrophic response to pressure overload in mice, demonstrating the importance of mTOR in regulating hypertrophic growth in vivo. ${ }^{36}$ This functional identity revealed by different bioinformatic interpretation confirmed that miRNAs have regulatory effects on cardiomyocyte hypertrophy by affecting signaling pathways.

Enrichment ranking of both signaling pathways and GOs indicated MAPK signaling pathway to be the most enriched. The miRNAs-gene interaction network analysis further integrated the GO analysis and KEGG findings, and then outlined the major targets of miRNAs. Members of the MAPK family had the highest ratio and enrichment in the MAPK signaling pathway. The MAPK pathways contain critical members of ERK1/2, c-Jun JNK, and p38, and serve as pivotal transducers of diverse biological functions including cell growth, differentiation, proliferation, and apoptosis. ${ }^{37,38}$ To validate the role of JNK, ERK1/2, and p38 in hyperglycemia-induced cardiac hypertrophy and glucose-induced cardiomyocyte hypertrophy, biological validation experiments were conducted. Western blot analysis demonstrated that all three critical members of MAPK cascades were activated in diabetic mice (hypertrophy stage). We further confirmed the role of MAPK pathway in neonatal rat cardiomyocyte hypertrophy model induced by high glucose. Both in vivo and in vitro results suggest that ERK1/2, JNK, and p38 are activated in the cardiac tissues of diabetic mice characterized by cardiac hypertrophy and in cultured neonatal cardiomyocyte hypertrophy induced by high glucose. Thum et $\mathrm{al}^{39}$ reported that miRNA-21 contributes to myocardial disease by stimulating MAP kinase signaling in fibroblasts. In addition, this group has also provided strong evidence that upregulation of miR-21 in cardiac hypertrophy is mediated by an increase in miR-21 expression in cardiac fibroblasts but not cardiomyocytes. These findings suggest that a possible reason as to why we did not observe the role of miR-21 in context of cardiomyocyte hypertrophy in diabetes.

To validate computational results, systems biological approaches are performed provide insight into the principles of the regulation of signaling networks by miRNAs. Previous work indicated that miR-373 plays an important role in invasion and metastasis of several malignancies, 
such as breast cancer, ${ }^{40}$ prostate cancer, ${ }^{41}$ testicular germ cell tumor, ${ }^{42}$ esophageal cancer, ${ }^{43}$ and hilar cholangiocarcinoma. ${ }^{44}$ At present, there is no report on how the miR-373 responds to diabetic cardiomyopathy. In our study, we found that miR-373 expression was decreased in the diabetic heart. To further explore the function of miR-373 in the regulation of glucose-induced cardiomyocyte hypertrophy, miR-373 was specifically up-regulated in neonatal rat cardiomyocytes. Exposure of neonatal rat cardiomyocytes to glucose and transfection with miR-373 mimic showed increased expression of miR-373 and cell size, suggesting a strong involvement of miR373 in the glucose-induced cardiomyocyte hypertrophy. Bioinformatics analysis indicates that MEF2C is a potential target gene of miR-373. MEF2C, a transcription factor, is involved in transcriptional regulation in postnatal hearts. ${ }^{45}$ Previous studies have demonstrated that glucose-induced cardiomyocyte hypertrophy is associated with increased activity of MEF2. ${ }^{14}$ To confirm this, the protein level of MEF2C was determined by Western blot. The results showed that upregulation of miR-373 decreased MEF2C expression in cultured cardiac myocytes, which suggested that MEF2C was a target gene of miR-373. To further determine whether MAPK signaling pathway is a downstream target of miR-373 that is involved in a mediated effect on cardiac myocytes, we tested whether up-regulation of miR-373 is able to influence p38 MAPK, ERK1/2, and JNK levels. As a result, transfection with miR-373 mimic did not alter levels of p38 MAPK, ERK1/2, or JNK. It appears that MAPK are not the downstream targets of miR-373. Previous study showed that p38 can directly regulate MEF2 transcription factors in hypertrophied heart. ${ }^{46,47}$ These findings encouraged us to further explore the relationship between MAPK pathway and miR-373 in glucose-induced cardiomyocyte hypertrophy. To this end, we examine whether blockade of p38 MAPK, ERK1/2, or ERK with specific inhibitors is sufficient to increase miR-373 levels. Our data demonstrate that inhibition of p38 MAPK potently decreased cell size and reduced miR-373 expression. However, the ERK $1 / 2$ and JNK inhibitors had no effect on miR-373 levels. It was indicated that p38 MAPK regulates miR-373 expression but not ERK $1 / 2$ and JNK. Inhibition of p38 MAPK also could lead to a reduction in levels of MEF2C. It is possible that p38 MAPK initiates high-glucose-induced cardiomyocyte hypertrophy by regulating miR373-dependent repression of MEF2C.

In conclusion, this study attempted to create a holistic view of diabetic heart tissue-specific miRNA expression profiles and predicted miRNA target genes using online databases. By integrating the GO analysis and KEGG pathway data obtained from function enrichment and pathway enrichment of tissue-specific miRNA targets, we obtained regulatory networks mediated by tissue-specific miRNAs. The current study also reveals that miR-373 is transcriptionally regulated by p38 MAPK. miR-373 protects against the high-glucose-induced cardiomyocyte hypertrophy by targeting the hypertrophic protein, MEF2C. Our findings suggest that miRNAs plays an important role in hyperglycemia-induced hypertrophy in cardiomyocytes through several signaling pathways, es- pecially MAPK signaling pathways, leading to myocardial dysfunction in diabetes. These findings provide information for future investigations into the mechanisms and pathology of diabetic cardiomyopathy.

\section{References}

1. Bartel DP: MicroRNAs: genomics, biogenesis, mechanism, and function. Cell 2004, 116:281-297

2. Plasterk RH: Micro RNAs in animal development. Cell 2006, 124 877-881

3. van Rooij E, Olson EN: MicroRNAs: Powerful new regulators of heart disease and provocative therapeutic targets. J Clin Invest 2007, 117:2369-2376

4. Divakaran V, Mann DL: The emerging role of microRNAs in cardiac remodeling and heart failure. Circ Res 2008, 103:1072-1083

5. Hu S, Huang M, Li Z, Jia F, Ghosh Z, Lijkwan MA, Fasanaro P, Sun N, Wang $X$, Martelli F, Robbins RC, Wu JC: MicroRNA-210 as a novel therapy for treatment of ischemic heart disease. Circulation 2010, 122:S124-S131

6. van Rooij E, Sutherland LB, Thatcher JE, DiMaio JM, Naseem RH, Marshall WS, Hill JA, Olson EN: Dysregulation of microRNAs after myocardial infarction reveals a role of miR-29 in cardiac fibrosis. Proc Natl Acad Sci USA 2008, 105:13027-13032

7. Yang B, Lin H, Xiao J, Lu Y, Luo X, Li B, Zhang Y, Xu C, Bai Y, Wang $H$, Chen $G$, Wang Z: The muscle-specific microRNA miR-1 regulates cardiac arrhythmogenic potential by targeting GJA1 and KCNJ2. Nat Med 2007, 13:486-491

8. Cheng Y, Ji R, Yue J, Yang J, Liu X, Chen H, Dean DB, Zhang C: MicroRNAs are aberrantly expressed in hypertrophic heart: Do they play a role in cardiac hypertrophy?. Am J Pathol 2007, 170:18311840

9. van Rooij E, Sutherland LB, Liu N, Williams AH, McAnally J, Gerard $\mathrm{RD}$, Richardson JA, Olson EN: A signature pattern of stress-responsive microRNAs that can evoke cardiac hypertrophy and heart failure. Proc Natl Acad Sci USA 2006, 103:18255-18260

10. Rubler S, Dlugash J, Yuceoglu YZ, Kumral T, Branwood AW, Grishman A: New type of cardiomyopathy associated with diabetic glomerulosclerosis. Am J Cardiol 1972, 30:595-602

11. Hayat SA, Patel B, Khattar RS, Malik RA: Diabetic cardiomyopathy: mechanisms, diagnosis and treatment. Clin Sci (Lond) 2004, 107: $539-557$

12. Khavandi K, Khavandi A, Asghar O, Greenstein A, Withers S, Heagerty AM, Malik RA: Diabetic cardiomyopathy-a distinct disease? Best Pract Res Clin Endocrinol Metab 2009, 23:347-360

13. Li J, Zhu H, Shen E, Wan L, Arnold JM, Peng T: Deficiency of rac1 blocks NADPH oxidase activation, inhibits endoplasmic reticulum stress, and reduces myocardial remodeling in a mouse model of type 1 diabetes. Diabetes 2010, 59:2033-2042

14. Feng B, Chen S, Chiu J, George B, Chakrabarti S: Regulation of cardiomyocyte hypertrophy in diabetes at the transcriptional level. Am J Physiol Endocrinol Metab 2008, 294:E1119-E1126

15. Kuo WW, Chung LC, Liu CT, Wu SP, Kuo CH, Tsai FJ, Tsai CH, Lu MC, Huang CY, Lee SD: Effects of insulin replacement on cardiac apoptotic and survival pathways in streptozotocin-induced diabetic rats. Cell Biochem Funct 2009, 27:479-487

16. Feng B, Chen S, George B, Feng Q, Chakrabarti S: miR133a regulates cardiomyocyte hypertrophy in diabetes. Diabetes Metab Res Rev 2010, 26:40-49

17. Shen E, Li Y, Shan L, Zhu H, Feng Q, Arnold JM, Peng T: Rac1 is required for cardiomyocyte apoptosis during hyperglycemia. Diabetes 2009, 58:2386-2395

18. Barbosa ME, Alenina N, Bader M: Induction and analysis of cardiac hypertrophy in transgenic animal models. Methods Mol Med 2005, 112:339-352

19. Zou Y, Hiroi Y, Uozumi H, Takimoto E, Toko H, Zhu W, Kudoh S, Mizukami M, Shimoyama M, Shibasaki F, Nagai R, Yazaki Y, Komuro I: Calcineurin plays a critical role in the development of pressure overload-induced cardiac hypertrophy. Circulation 2001, 104:97-101

20. Livak KJ, Schmittgen TD: Analysis of relative gene expression data using real-time quantitative PCR and the 2(-Delta Delta $\mathrm{C}(\mathrm{T})$ ) method. Methods 2001, 25:402-408 
21. Sam LT, Mendonca EA, Li J, Blake J, Friedman C, Lussier YA: PhenoGO: an integrated resource for the multiscale mining of clinical and biological data. BMC Bioinformatics 2009, 10 Suppl 2:S2-S8

22. Sayed D, Hong C, Chen IY, Lypowy J, Abdellatif M: MicroRNAs play an essential role in the development of cardiac hypertrophy. Circ Res 2007, 100:416-424

23. Callis TE, Pandya K, Seok HY, Tang RH, Tatsuguchi M, Huang ZP, Chen JF, Deng Z, Gunn B, Shumate J, Willis MS, Selzman CH, Wang DZ: MicroRNA-208a is a regulator of cardiac hypertrophy and conduction in mice. J Clin Invest 2009, 119:2772-2786

24. Fang ZY, Prins JB, Marwick TH: Diabetic cardiomyopathy: Evidence, mechanisms, and therapeutic implications. Endocr Rev 2004, 25: 543-567

25. Dolan ME, Ni L, Camon E, Blake JA: A procedure for assessing GO annotation consistency. Bioinformatics 2005, 21 Suppl 1:i136-i143

26. Draghici S, Khatri P, Tarca AL, Amin K, Done A, Voichita C, Georgescu C, Romero R: A systems biology approach for pathway level analysis. Genome Res 2007, 17:1537-1545

27. Yi M, Horton JD, Cohen JC, Hobbs HH, Stephens RM: WholePathwayScope: A comprehensive pathway-based analysis tool for high-throughput data. BMC Bioinformatics 2006, 7:1-24

28. Kanehisa M, Goto S, Kawashima S, Okuno Y, Hattori M: The KEGG resource for deciphering the genome. Nucleic Acids Res 2004, 32: D277-D280

29. Esposito G, Prasad SV, Rapacciuolo A, Mao L, Koch WJ, Rockman $\mathrm{HA}$ : Cardiac overexpression of a $\mathrm{G}(\mathrm{q})$ inhibitor blocks induction of extracellular signal-regulated kinase and c-Jun $\mathrm{NH}(2)$-terminal kinase activity in in vivo pressure overload. Circulation 2001, 103:1453-1458

30. Ruwhof C, van der Laarse A: Mechanical stress-induced cardiac hypertrophy: Mechanisms and signal transduction pathways. Cardiovasc Res 2000, 47:23-37

31. Malekar P, Hagenmueller M, Anyanwu A, Buss S, Streit MR, Weiss CS, Wolf D, Riffel J, Bauer A, Katus HA, Hardt SE: Wnt signaling is critical for maladaptive cardiac hypertrophy and accelerates myocardial remodeling. Hypertension 2010, 55:939-945

32. Parker TG, Packer SE, Schneider MD: Peptide growth factors can provoke "fetal" contractile protein gene expression in rat cardiac myocytes. J Clin Invest 1990, 85:507-514

33. Shimojo N, Jesmin S, Zaedi S, Otsuki T, Maeda S, Yamaguchi N, Aonuma K, Hattori Y, Miyauchi T: Contributory role of VEGF overexpression in endothelin-1-induced cardiomyocyte hypertrophy. Am J Physiol Heart Circ Physiol 2007, 293:H474-H481

34. Pan J, Fukuda K, Kodama H, Makino S, Takahashi T, Sano M, Hori S, Ogawa S: Role of angiotensin II in activation of the JAK/STAT pathway induced by acute pressure overload in the rat heart. Circ Res 1997 , 81:611-617
35. Pan J, Fukuda K, Kodama H, Sano M, Takahashi T, Makino S, Kato T, Manabe T, Hori S, Ogawa S: Involvement of gp130-mediated signaling in pressure overload-induced activation of the JAK/STAT pathway in rodent heart. Heart Vessels 1998, 13:199-208

36. McMullen JR, Sherwood MC, Tarnavski O, Zhang L, Dorfman AL, Shioi T, Izumo S: Inhibition of mTOR signaling with rapamycin regresses established cardiac hypertrophy induced by pressure overload. Circulation 2004, 109:3050-3055

37. Molkentin JD, Dorn GW, 2nd: Cytoplasmic signaling pathways that regulate cardiac hypertrophy. Annu Rev Physiol 2001, 63:391-426

38. Muslin AJ: MAPK signalling in cardiovascular health and disease: Molecular mechanisms and therapeutic targets. Clin Sci (Lond) 2008 , 115:203-218

39. Thum T, Gross C, Fiedler J, Fischer T, Kissler S, Bussen M, Galuppo P, Just S, Rottbauer W, Frantz S, Castoldi M, Soutschek J, Koteliansky V, Rosenwald A, Basson MA, Licht JD, Pena JT, Rouhanifard SH, Muckenthaler MU, Tuschl T, Martin GR, Bauersachs J, Engelhardt S: MicroRNA-21 contributes to myocardial disease by stimulating MAP kinase signalling in fibroblasts. Nature 2008, 456:980-984

40. Yan GR, Xu SH, Tan ZL, Liu L, He QY: Global identification of miR373-regulated genes in breast cancer by quantitative proteomics. Proteomics 2011, 11:912-920

41. Yang K, Handorean AM, Iczkowski KA: MicroRNAs 373 and 520c are downregulated in prostate cancer, suppress CD44 translation and enhance invasion of prostate cancer cells in vitro. Int J Clin Exp Pathol 2009, 2:361-369

42. Voorhoeve PM, le Sage C, Schrier M, Gillis AJ, Stoop H, Nagel R, Liu YP, van Duijse J, Drost J, Griekspoor A, Zlotorynski E, Yabuta N, De Vita G, Nojima H, Looijenga LH, Agami R: A genetic screen implicates miRNA-372 and miRNA-373 as oncogenes in testicular germ cell tumors. Cell 2006, 124:1169-1181

43. Lee KH, Goan YG, Hsiao M, Lee CH, Jian SH, Lin JT, Chen YL, Lu PJ: MicroRNA-373 (miR-373) post-transcriptionally regulates large tumor suppressor, homolog 2 (LATS2) and stimulates proliferation in human esophageal cancer. Exp Cell Res 2009, 315:2529-2538

44. Chen Y, Gao W, Luo J, Tian R, Sun H, Zou S: Methyl-CpG binding protein MBD2 is implicated in methylation-mediated suppression of miR-373 in hilar cholangiocarcinoma. Oncol Rep 2010, 25:443-451

45. Kolodziejczyk SM, Wang L, Balazsi K, DeRepentigny Y, Kothary R, Megeney LA: MEF2 is upregulated during cardiac hypertrophy and is required for normal post-natal growth of the myocardium. Curr Biol 1999, 9:1203-1206

46. Han J, Molkentin JD: Regulation of MEF2 by p38 MAPK and its implication in cardiomyocyte biology. Trends Cardiovasc Med 2000 10:19-22

47. Akazawa $\mathrm{H}$, Komuro I: Roles of cardiac transcription factors in cardiac hypertrophy. Circ Res 2003, 92:1079-1088 